\title{
Monitoring hydration process and quality of sand grouted with microfine-cement using shear wave velocity and electrical conductivity measurements
}

\author{
Hyunwook Choo ${ }^{1, *}$, Hongyeop $\mathrm{Nam}^{2}$, Changho Lee ${ }^{3}$, Woojin $\mathrm{Lee}^{2}$, and Susan Burns ${ }^{4}$ \\ ${ }^{1}$ Kyung Hee University, Department of Civil Engineering, 17104 Yongin, South Korea \\ ${ }^{2}$ Korea University, School of Civil, Environmental, and Architectural Engineering, 02841 Seoul, South Korea \\ ${ }^{3}$ Jonnam National University, Department of Marine Civil Engineering, 59626 Yeosu, South Korea \\ ${ }^{4}$ Georgia Institute of Technology, School of Civil and Environmental Engineering, 30332 Atlanta, GA, USA
}

\begin{abstract}
This study aims at monitoring the grouting status such as hydration process and quality of sand grouted with microfine-cement using shear wave velocity $\left(V_{s}\right)$ and electrical conductivity (EC) measurement techniques. The microfine-cement with the maximum particle size $<15 \mu \mathrm{m}$ and the three angular sands with median particle sizes ranging from $0.47 \mathrm{~mm}$ to $1.01 \mathrm{~mm}$ were used in this study. The testing specimens were prepared at relative density $\sim 70 \%$ and water to cement ratio $=1.0,1.5$, and 2.0 in a split plastic mold for the real time continuous measurements of $V_{s}$ and EC, and for the unconfined compression tests at the end of curing time of 3, 7, 14, and 28 days. The results demonstrate that both unconfined compressive strength (UCS) and $V_{s}$ show almost constant values after the curing period of 20-28 days; while, the variation of EC is negligible after the curing period of 10-20 days. Therefore, the shear wave velocity measurement technique can be superior to electrical conductivity measurement in terms of monitoring of hydration process of tested sands grouted with microfine-cement. However, both $V_{s}$ and EC show strong linear relationship with UCS of tested materials, reflecting both non-destructive testing methods can be beneficially used for the quality evaluation of grouted materials.
\end{abstract}

\section{Introduction}

The permeation grouting technique with microfinecement is an efficient method for enhancing strength and stiffness of the soil that Ordinary Portland cement grout fails to. The unconfined compression (UC) test through coring or boring is the most common testing method for the evaluation of reinforcement effect of soft ground grouted with cementing materials. However, UC test requires a lot of cost and time to prepare testing specimens, and the specimens for UC test can be disturbed during the sampling process [1-6]. Thus, this study aims at monitoring the grouting status such as hydration process and quality of sand grouted with microfine-cement using non-destructive testing methods (i.e., shear wave velocity and electrical conductivity measurement techniques).

\section{Experimental Program}

To carry out UC test, bender element test, and electrical conductivity measurement test, three sands with varying median particle sizes were used (Table 1). To prepare cement-grouted specimens, the commercially available microfine-cement (Ssangyong Company, South Korea) with the maximum particle size $<15 \mu \mathrm{m}$ and specific surface area $>860 \mathrm{~m}^{2} / \mathrm{kg}$ was used. Three different water-to-cement $(\mathrm{W} / \mathrm{C})$ ratios $(1,1.5$ and 2$)$ were selected in grouting process, and the testing specimens were prepared at relative density $\sim 70 \%$ in a split plastic mold for the real time continuous measurements of shear wave velocity $\left(V_{s}\right)$ and electrical conductivity (EC), and for the UC tests at the end of curing time of $3,7,14$, and 28 days.

Table 1. Material properties.

\begin{tabular}{|c|c|c|c|c|}
\hline Type & $G_{s}$ & $D_{50}(\mathrm{~mm})$ & $e_{\max }$ & $e_{\min }$ \\
\hline $\mathrm{K}-4$ & 2.65 & 1.01 & 1.08 & 0.71 \\
\hline $\mathrm{K}-5$ & 2.65 & 0.80 & 1.07 & 0.69 \\
\hline $\mathrm{K}-6$ & 2.65 & 0.47 & 1.03 & 0.66 \\
\hline
\end{tabular}

Note: $G_{s}=$ specific gravity; $D_{50}=$ median particle size; $e_{\max }$ and $e_{\min }=$ maximum and minimum void ratios, respectively

An unconfined compression test was performed using a Universal Testing Machine with a capacity of 20 $\mathrm{kN}$ at a loading rate of $1 \mathrm{~mm} / \mathrm{min}$ at the end of each curing period (i.e., 3, 7, 14, and 28 days). The top and bottom of the testing specimens were covered with an unbonded cap to apply uniform stress. To measure the $V_{s}$, bender element tests were performed. A $20 \mathrm{HZ}$ and $10 \mathrm{~V}$ square wave was generated by the source bender element, which is connected to a function generator

\footnotetext{
* Corresponding author: choohw@khu.ac.kr
} 
(Agilent, 34970A). The shear wave propagated through the soil specimen was detected by the receiver bender element. The signals from the receiver were filtered and amplified by a filter amplifier (Krohn-Hite, 3364). And then, the received signals were digitized by an oscilloscope (Agilent, 54624A). The distance of wave propagation is calculated from tip-to-tip distance between bender elements. The stacking method that a total signal of 1024 were used for eliminate random noise. In order to evaluate an electrical conductivity of grouted sand, four electrodes system was employed in this study. During the experiment, electrical current flowed between two current electrodes and the electrical resistance were measured by the two potential electrodes using an LCR meter. The frequency of the LCR meter was selected to be $10 \mathrm{kHz}$ to avoid electrode polarization and electrical resonance, and input voltage was $1 \mathrm{~V}$. From the measured electrical resistance and the relevant calibration factor, the EC was calculated.

\section{Results and Discussion}

\subsection{Unconfined compressive strength}

After grouting, the unconfined compressive strength (UCS) increases due to hydration over time, and the increasing rate of UCS over time decreases with an increase in time. Therefore, after a certain period of time, a nearly constant UCS value can be observed. Figure 1 demonstrates that UCS of the tested materials shows an almost constant value after 20-28 days. Because the change of UCS with time may represent the progress of the hydration process, Figure 1 reveals that the hydration process may be completed after about 20-28 days for the grouting sample used in this test.

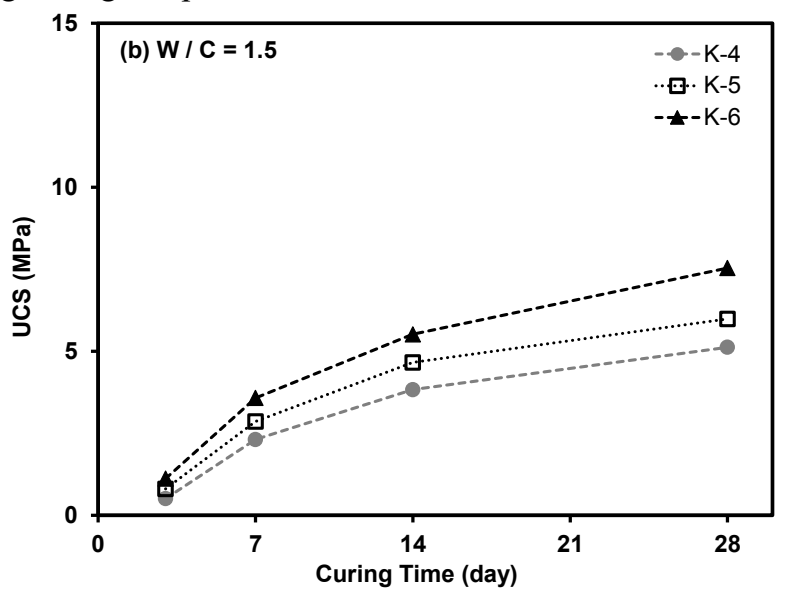

Fig. 1. Variation of UCS with curing time.

One of the most important variable determining the UCS of grouted sand is UCS of pure grout or W/C of grout [2, 7-9]. Figure 2 shows the variation of UCS as a function of $\mathrm{W} / \mathrm{C}$ for all tested specimens at relative density $\sim 70 \%$ at 28 days of curing. The UCS of the specimen increases exponentially with a decrease in $\mathrm{W} / \mathrm{C}$ because the strength of the cementation contact bonds between sand particles can be increased with decreasing $\mathrm{W} / \mathrm{C}[3,10,11]$. Additionally, it can be observed in Figure 2 that UCS of tested materials generally increases with decreasing median particle size of sand because of an increase in specific surface with a decrease in particle size: sand particles with larger specific surface can effectively adsorb the cement particles.

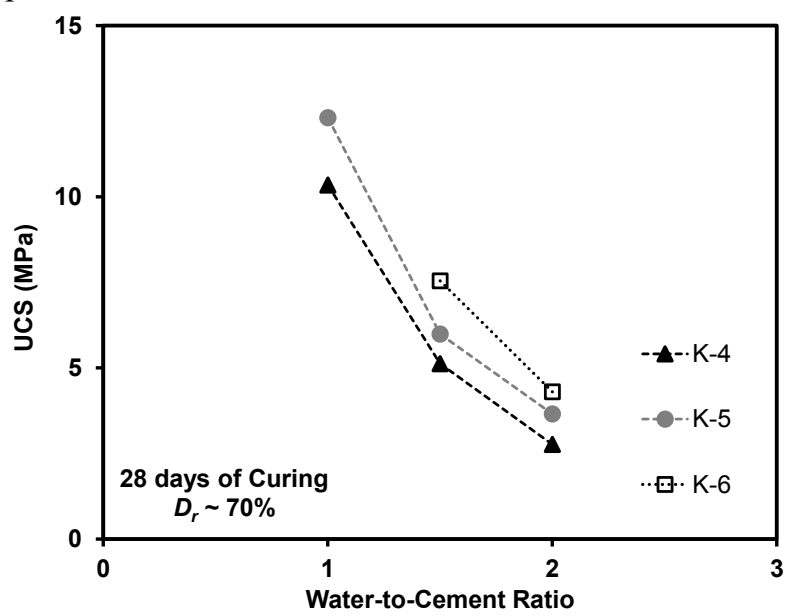

Fig. 2. Dependency of UCS on water-to-cement ratio.

\subsection{Maximum Shear Modulus}

Figure 3 shows the maximum shear modulus $\left(G_{\max }\right)$ of grouted sand at $\mathrm{W} / \mathrm{C}=1.5$ according to curing period. Note that the $G_{\max }$ was calculated based on the following simple equation: $G_{\max }=\rho V_{s}^{2}$, where $\rho=$ mass density. It can be observed in Figure 4 that the $G_{\max }$ of grouted sand increases with curing time because the grout is hydrated and the bonds become stronger with time. (Maher et al 1994; Dano and Hicher 2002). Similar to the results for UCS (Figure 1), an almost constant $G_{\max }$ values can be observed after 20 days, which is slightly earlier than the required time for an almost constant UCS values.

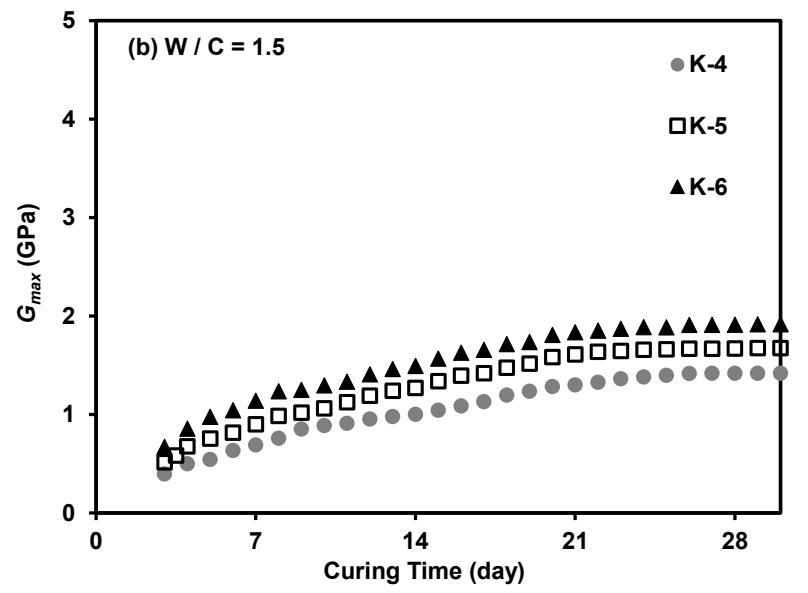

Fig. 3. Variation of $G_{\max }$ with curing time.

Figure 3 also demonstrates that $G_{\max }$ of tested materials generally increases with decreasing median particle size of sand because of an increase in specific surface with a decrease in particle size. This lead to an increased number of cementation bonds between the sand particles with decreasing sand particle size $[12,13]$. The decrease in sand particle size thus results in a better cementation effect, and a consequent increase in $G_{\max }$. 


\subsection{Electrical Conductivity (or Resistivity)}

Figure 4 shows the variation of the measured electrical resistivity (ER) of tested materials at $\mathrm{W} / \mathrm{C}=1.5$ as a function of curing time. Similar to the results of UCS and $G_{\max }$ (Figures 1 and 3), the measured ER is affected by median particle size of sand. In addition, an almost constant ER can be observed after a certain period of curing time, which is similar to the results of UCS and $G_{\max }$. However, the variation of ER at early curing stage is very different from that of UCS or $G_{\max }$ : (1) initially, ER slightly decreases with time; (2) ER very slowly increases with time ( $<10$ days of curing); (3) ER rapidly increases with time after around 10 days of curing.

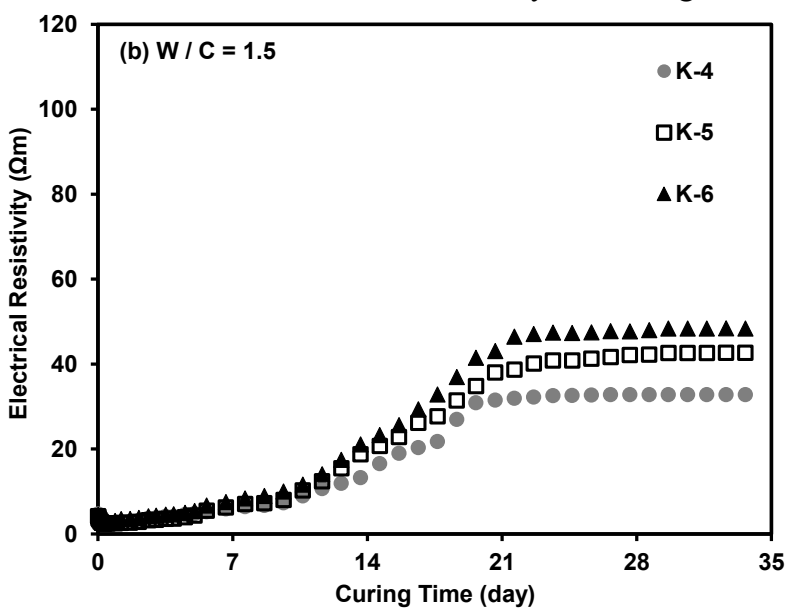

Fig. 4. Variation of electrical resistivity with curing time.

To highlight the variation of electrical properties of the tested materials at an early curing stage, the variation of EC $(=1 / \mathrm{ER})$ is plotted as a function of curing time in $\log$ scale (Figure 5). The EC of cemented materials is mainly determined by pore water conduction, which is the ionic conduction through the pore space between solid particles. Note that pore water conduction is mainly determined by pore water conductivity, porosity, and tortuosity. At a very early curing stage, the mobile ions such as $\mathrm{Na}^{+}, \mathrm{K}^{+}, \mathrm{SO}^{2+}, \mathrm{OH}^{-}$and $\mathrm{Ca}^{2-}$ can be dissolved from cement pastes, leading to an increase in pore water conductivity. Therefore, the measured EC at very early curing stage can increase with time. However, with an increase in curing time, the hydration products such as calcium silicate hydrate gel $(\mathrm{C}-\mathrm{S}-\mathrm{H})$ and ettringite can be formed. This results in a decrease in porosity and an increases in the tortuosity of pore network. Consequently, the EC rapidly decrease with time, and approach to the constant value at later curing stage $(>10$ days).

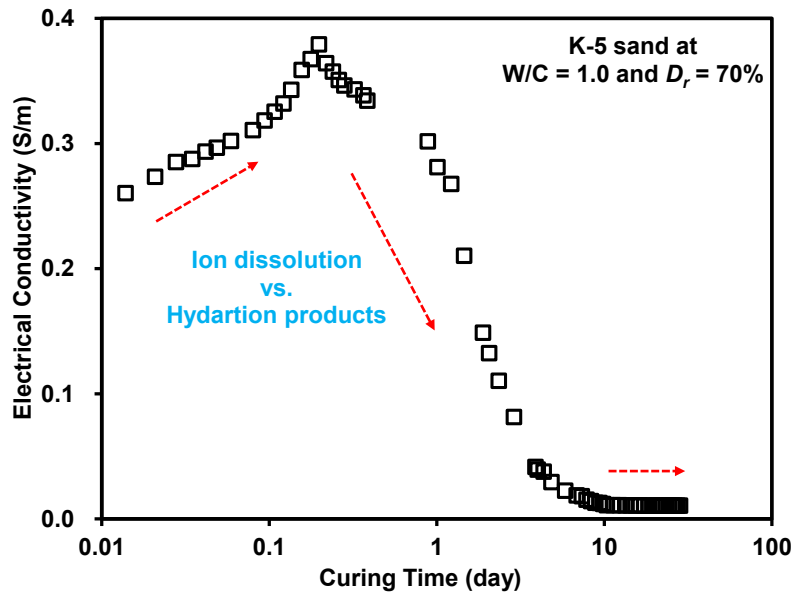

Fig. 5. Variation of electrical conductivity with curing time.

\subsection{Monitoring of Curing Process}

In order to monitor the hydration process through the non-destructive tests (shear wave velocity measurement and electrical conductivity measurement), the timedependent $V_{s}$ and EC measurement tests were performed on the same sample for the UCS measurement. The variations of UCS, $G_{\max }$, and ER of tested materials for $\mathrm{W} / \mathrm{C}=1.0$ according to curing time are plotted in Figure 6 . Because the variations of UCS, $G_{\max }$, and ER with time show nonlinear behaviour, the hyperbolic model was employed to estimate the time-dependent variation of UCS, $G_{\max }$, and ER. Note that the estimated UCS, $G_{\max }$, and ER based on hyperbolic model are also included in Figure 6.

The UCS and $G_{\max }$ of grouted sand is mainly determined by the strength and stiffness of cementation bonding between sand particles, respectively. Due to the difference in strain levels or inherent difference between strength and stiffness, the time required for an almost constant UCS is slightly greater than that for an almost constant $G_{\max }$. However, because both UCS and $G_{\max }$ are determined by the quality of cementation bondings, which is determined by the hydration process, UCS and $G_{\max }$ of tested materials show comparable timedependent behaviours. Therefore, both UCS and $G_{\max }$ can be greatly captured by the hyperbolic model (Figure 6). However, Figure 6 (c) demonstrates that the variation of ER according to curing time cannot adequately capture by the hyperbolic model, reflecting the fundamental difference between UCS (or $G_{\max }$ ) and ER. Because the ER of grouted soils is mainly determined by the pore water conduction as mentioned earlier, the mobile ions and free water are essential for the transport of ions. Due to the offset mechanism between the increase in the concentration of mobile ions and the decrease in the availability of free water at early curing stage, the variation of ER at early curing stage $(<10$ days) cannot be captured by hyperbolic model. In addition, Figure 6 demonstrates that the electric conductivity (EC) was almost constant after about 15-22 days, which is faster than the time required for almost constant values of UCS and/or $G_{\max }$. This is because UCS and $G_{\max }$ are determined by the quality of 
cementation bonding between sand particles, whereas EC is determined by the degree of freezing and the degree of pore clogging as hydration progresses.
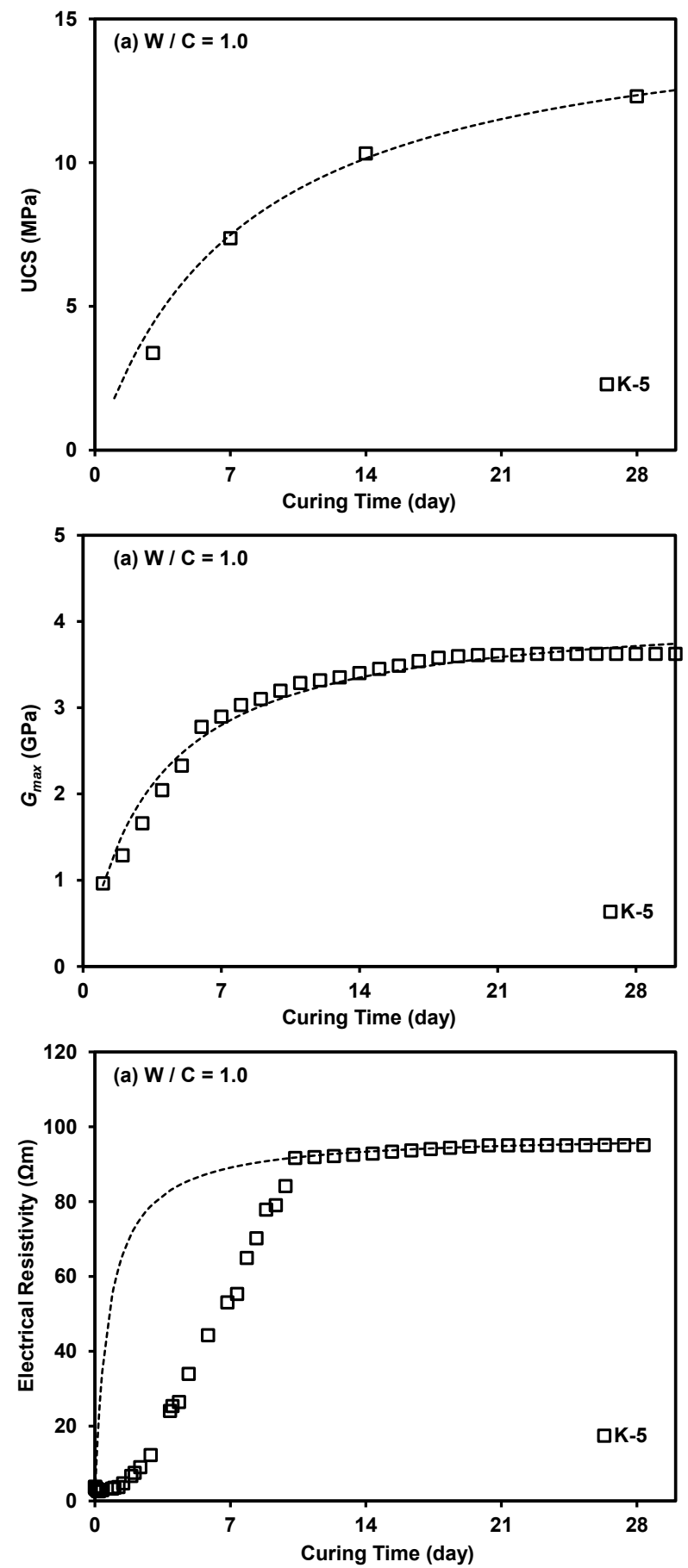

Fig. 6. Fitting the measured values with hyperbolic model: (a) UCS; (b) $G_{\max }$; and (c) electrical resistivity.

\subsection{Comparison between Different Measurements}

Figure 7 shows the relationship between UCS and $G_{\max }$ and UCS and EC. Because both UCS, $G_{\max }$, and EC vary by similar factors (e.g., sand particle size, W/C, and others), Figure 7 reveals that the UCS of grouted sands can be adequately estimated from the measurements of $G_{\max }$ and/or EC.
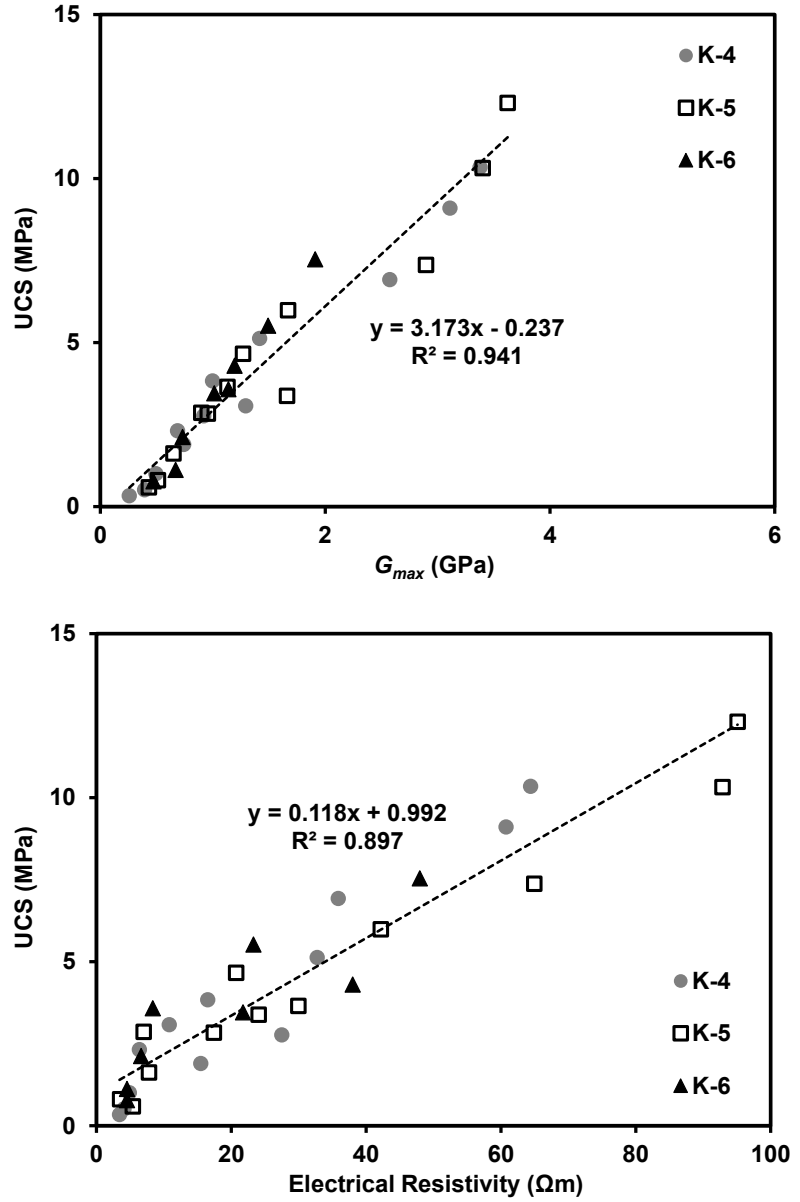

Fig. 7. Estimating UCS using (a) $G_{\max }$ and (b) electrical resistivity.

\section{Conclusion}

Compared with the electrical conductivity measurement, the hydration process can be accurately monitored through shear wave velocity measurement. In the case of the correlation with the uniaxial compressive strength, both the shear wave velocity (or shear modulus) and the electric conductivity showed good correlation, and it was confirmed that the uniaxial compressive strength prediction was possible through the non-destructive tests.

\section{References}

1. Kaga, M. and R. Yonekura, Soils and Foundations, 1991. 31(3): p. 43-59.

2. Dano, C., P.-Y. Hicher, and S. Tailliez, Journal of Geotechnical and Geoenvironmental Engineering, 2004. 130(3): p. 328-338.

3. Markou, I. and A. Droudakis, Geotechnical and Geological Engineering, 2013. 31(4): p. 1041-1058.

4. Sunitsakul, J., A. Sawatparnich, and A. Sawangsuriya, Geotechnical and Geological Engineering, 2012. 30(1): p. 263-268.

5. Avci, E. and M. Mollamahmutoğlu, Journal of Materials in Civil Engineering, 2016. 28(12): p. 06016015 . 
6. Tinoco, J., A.G. Correia, and P. Cortez, Construction and Building Materials, 2011. 25(3): p. 1257-1262.

7. Zebovitz, S., R. Krizek, and D. Atmatzidis, Journal of Geotechnical Engineering, 1989. 115(12): p. 1717-1733.

8. Schwarz, L.G. and R.J. Krizek, Geotechnical Testing Journal, 1994. 17(4): p. 434-443.

9. Schwarz, L.G. and M. Chirumalla, Innovative concepts and applications. 2007. p. 1-15.

10. Choo, H., H. Nam, and W. Lee, Journal of Applied Geophysics, 2017. 147: p. 102-108.

11. Choo, H., W. Lee, and C. Lee, Geotechnical Testing Journal, 2018. 41, no. 5.

12. Ismail, M.A., et al., Geotechnique, 2002. 52(5): p. 313-324.

13. Yang, L. and L. Salvati. the Fifth International Conference on Recent Advances in Geotechnical Earthquake Engineering and Soil Dynamics and Symposium in Honor of Professor I.M. Idriss. 2010. San Diego, Calif. 